\title{
Phenylethanolamine N-Methyltransferase Gene Polymorphisms and Adverse Outcomes in Acute Kidney Injury
}

\author{
Ahsan Alam $^{a}$ Daniel T. O'Connor ${ }^{c}$ Mary C. Perianayagam ${ }^{b}$ Alexey Y. Kolyada $^{b}$ \\ Yuqing Chen ${ }^{c}$ Fangwen Raoc Manjula Mahatac Sushil Mahatac \\ Orfeas Liangos $^{\mathrm{b}}$ Bertrand L. Jaber ${ }^{\mathrm{b}}$ \\ ${ }^{a}$ Division of Nephrology, Tufts Medical Center and ${ }^{b}$ Division of Nephrology, Kidney and Dialysis Research \\ Laboratory, St. Elizabeth's Medical Center, Boston, Mass., and ' Department of Medicine and Center for Human \\ Genetics and Genomics, University of California at San Diego, La Jolla, Calif., USA
}

\section{Key Words}

Acute kidney injury $\cdot$ Phenylethanolamine

$\mathrm{N}$-methyltransferase $\cdot$ Single nucleotide polymorphisms • Mortality

\begin{abstract}
Background/Aims: The catecholaminergic pathway is important in the physical stress response; however, its role is not well understood in acute kidney injury (AKI). We studied single nucleotide polymorphisms (SNPs) of phenylethanolamine $\mathrm{N}$-methyltransferase (PNMT), the terminal enzyme of the catecholaminergic pathway, and their association with adverse outcomes in AKI. Methods: We performed a casecontrol study of 961 Caucasian subjects (194 with AKI and 767 controls). The PNMT promoter G-161A (rs876493) and coding A+1543G (rs5638) SNPs were genotyped and haplotypes generated. The outcomes of interest were the development of AKI, in-hospital mortality, dialysis requirement, oliguria, and hemodynamic shock. Urine catecholamines were measured in cases to explore genotype-phenotype correlations. Results: The PNMT $+1543 \mathrm{G}$ allele was associated with AKI [odds ratio (OR) 2.19,95\% confidence interval (CI): 1.04-4.60]. For AKI cases, each PNMT -161 A allele was
\end{abstract}

associated with lower mortality (OR $0.58,95 \% \mathrm{Cl}: 0.35-0.99)$ and hemodynamic shock (OR $0.63,95 \% \mathrm{Cl}: 0.40-1.00)$. The PNMT $+1543 \mathrm{G}$ allele was associated with oliguria (OR 3.35, 95\% Cl: 1.13-9.95). Urine adrenaline was associated with increased hemodynamic shock and mortality, but was lowest in PNMT -161 A/A carriers. Conclusion: In Caucasians, PNMT SNPs are associated with the development of AKI, disease severity, and in-hospital mortality. The adrenergic pathway provides another area of focus in the study of AKI.

Copyright $\odot 2010$ S. Karger AG, Basel

\section{Introduction}

Acute kidney injury (AKI) is an important and complex disorder associated with significant morbidity and mortality. The incidence of AKI in hospitalized patients is estimated to be $5-10 \%$, and is much higher in the critically ill [1-3]. AKI, like any other acute physiologic stress,

Results presented in this paper have not been published previously in whole or part, except in abstract format at the 2008 American Society of Nephrology annual meeting in Philadelphia, Pa., USA.

\section{KARGER}

() 2010 S. Karger AG, Basel

Fax +41613061234 E-Mail karger@karger.ch www.karger.com www.karger.com/nec
Bertrand L. Jaber, MD, MS

Department of Medicine, St. Elizabeth's Medical Center

736 Cambridge Street

Boston, MA 02135 (USA)

Tel. +1 617562 7832, Fax +1 617562 7797, E-Mail bertrand.jaber@ caritaschristi.org 
may evoke the biosynthesis and release of various adrenergic hormones [4]. Stress-regulated control of catecholamines, with epinephrine as the major stress hormone, may play an important role in the development, evolution, propagation, or resolution of AKI.

Physical stress results in epinephrine and norepinephrine release and the stimulation of the catecholamine biosynthetic enzymes, tyrosine hydroxylase, dopamine $\beta$ hydroxylase, and phenylethanolamine N-methyltransferase (PNMT) [5]. The biosynthetic activity of PNMT, the terminal enzyme of the catecholaminergic pathway, converts epinephrine from norepinephrine by N-methylation, thereby replenishing depleted stores $[4,6]$. Genetic single nucleotide polymorphism (SNP) analyses have commonly been employed in the study of complex disease traits. Adrenergic pathway gene SNPs may result in an altered homeostatic response during periods of acute illness. Little is known of their role in the context of patients with AKI.

Using a case-control design and a candidate gene approach, we first examined the association of 2 PNMT gene SNPs with the development of hospital-acquired AKI in an adult Caucasian population. Among those hospitalized with AKI, we prospectively explored the association of each SNP and their common haplotypes with the outcomes of in-hospital mortality, dialysis requirement, oliguria, and hemodynamic shock. Finally, the SNPs and AKI-related outcomes were related to the intermediate phenotype of urine adrenaline and noradrenaline levels.

\section{Subjects and Methods}

\section{Study Design and Population}

Hospitalized patients with AKI were recruited from 2 tertiary care hospitals in Boston, Mass., USA. Subjects were recruited from November 2003 to January 2007. All eligible patients were $\geq 18$ years of age and received in-hospital nephrology consultation for AKI. Subjects were excluded if they were pregnant, required chronic dialysis, had an organ transplant in the past 1 year, or any evidence of acute obstructive uropathy. Informed consent was obtained for each subject, and the Institutional Review Board at both hospitals approved the protocol.

In addition, we obtained data for a control group of 767 nonhospitalized adult Caucasian subjects who had been genotyped for the same 2 PNMT gene SNPs, but had no diagnosis of AKI. The mean ( \pm standard deviation) age of the control group was 45 \pm 17 years. Fifty-four percent were men and $19 \%$ had a diagnosis of hypertension.

\section{Data Collection}

Medical records were reviewed prospectively to retrieve data on the hospitalized patients with AKI including demographic characteristics, coexisting conditions, hospitalization course, and outcomes. AKI was defined at enrollment according to an increase in serum creatinine by 44,89 or $133 \mu \mathrm{mol} / 1(0.5,1.0$ or $1.5 \mathrm{mg} / \mathrm{dl}$ ) from a baseline level of $\leq 176,177-434$ or $\geq 435$ $\mu \mathrm{mol} / \mathrm{l}(\leq 1.9,2.0-4.9$ and $\geq 5.0 \mathrm{mg} / \mathrm{dl})$, respectively [7]. Stages of AKI were later classified according to the staging system proposed by the AKI Network [8]. Sepsis was ascertained using the systemic inflammatory response syndrome criteria [9]. For subjects with multiple biogeographic ancestries, self-identified ethnicity was used, and our analysis was restricted only to Caucasian study participants to account for population stratification.

\section{Genotyping Analyses}

DNA was prepared from blood leukocytes with an automated device (X-tractor Gene, Corbett Robotics, Brisbane, Qld., Australia), and samples were genotyped using a Pyrosequencing ${ }^{\mathrm{TM}}$ I96A (Pyrosequencing AB, Uppsala, Sweden) instrument according to standardized protocol [10]. Resulting sequences were analyzed by visual inspection and automatically by SNP software. The PNMT local genomic region was 'tagged' with 2 SNPs: proximal promoter G-161A (rs876493) and coding region (exon 3) A+1543G (synonymous Lys152Lys, rs5638). Base positions are numbered with respect to the cap (exon 1 start) site, in National Center for Biotechnology Information genomic (phage lambda vector) source clone X52730 [11]. These 2 SNPs were selected since they spanned both the promoter and the 2,210-bp coding region, and were nonredundant in that their pair-wise correlations were relatively low $\left(\mathrm{r}^{2}=0.02\right.$ in 23 control white subjects). Given the low degree of linkage disequilibrium between these 2 SNPs by SNP spectral decomposition, the effective number of SNPs tested was $\sim 1.98$. We maintained a $\mathrm{p}<0.05$ as the threshold for significance due to the exploratory nature of our analysis.

Haplotypes were generated from the 2 PNMT gene SNPs using a previously described statistical algorithm (Haplotype Resolution, Version 3.0) [12]. The 2 most common haplotype distributions were haplotype $1(-161 \mathrm{G} /+1543 \mathrm{~A})$ and haplotype $2(-161 \mathrm{~A} /$ $+1543 \mathrm{~A}$ ), and these were expressed as the number of chromosomal copies of the haplotype for each patient. The $-161 \mathrm{G} /+1543 \mathrm{G}$ haplotype did not exist in our cohort. We also created a diploid haplotype representing the number of copies of haplotypes 1 and 2 (haplotype 1/haplotype 1 homozygotes, haplotype 1/haplotype 2 heterozygotes, or haplotype 2 /haplotype 2 homozygotes).

\section{Urine Catecholamine Measurements}

Urine adrenaline and noradrenaline were measured in patients with AKI using a competitive ELISA (Labor Diagnostika Nord, Nordhorn, Germany). Adrenaline and noradrenaline were extracted from urine samples using a cis-diol-specific affinity gel, acylated and then derivatized enzymatically for quantification. The limit of detection for adrenaline and noradrenaline in urine are 0.33 and $1.33 \mathrm{ng} / \mathrm{ml}$, respectively. The intra-assay and interassay coefficients of variation were 11.0 and $14.3 \%$ for adrenaline, and 13.0 and $11.8 \%$ for noradrenaline. Catecholamine levels were normalized to creatinine excretion $(\mathrm{mg} / \mathrm{dl})$ from the same urine collection. A ratio of adrenaline to noradrenaline was also calculated. Due to markedly skewed distributions, catecholamine levels were log transformed for analysis.

\section{Outcome Measures}

The primary outcomes were all-cause in-hospital mortality, dialysis requirement during hospitalization, as well as the development of oliguria or hemodynamic shock. Dialysis requirement 
Table 1. Observed and expected distribution for each PNMT gene polymorphism in cases and controls

\begin{tabular}{lllllll}
\hline Locus & SNP & $\begin{array}{l}\text { Ref SNP No. Domain } \\
\text { (dbSNP) }\end{array}$ & Group & $\begin{array}{l}\text { Variant } \\
\text { allele }\end{array}$ & $\begin{array}{l}\text { HWE } \\
\text { p value }\end{array}$ \\
\hline PNMT & -161 G to A & rs876493 & promoter & $\begin{array}{l}\text { cases } \\
\text { controls }\end{array}$ & $\begin{array}{l}\text { A (50\%) } \\
\text { A (45\%) }\end{array}$ & $\begin{array}{l}0.14 \\
0.13\end{array}$ \\
\hline PNMT & +1543 A to G & rs5638 & exon 3 (Lys152Lys) & $\begin{array}{l}\text { cases } \\
\text { controls }\end{array}$ & G (4\%) & 0.55 \\
& & & & & & \\
\end{tabular}

HWE = Hardy-Weinberg equilibrium

was defined as the initiation of any intermittent or continuous dialysis therapy. Urine output was measured for a 24-hour period at study enrolment, and oliguria was defined as a urine volume $<400 \mathrm{ml} /$ day. Hemodynamic shock was defined by a systolic blood pressure $<100 \mathrm{~mm} \mathrm{Hg}$ and a heart rate $>100$ beats/min, or the use of vasopressor agents.

\section{Statistical Analysis}

Genotype frequencies for each PNMT gene polymorphism were examined for deviation from Hardy-Weinberg equilibrium using the $\chi^{2}$ test. Comparisons across genotypes were performed for each covariate of interest. Data are presented as proportions, means and standard deviations, or as medians with interquartile ranges, as appropriate. The Student t or analysis of variance tests were used for normally distributed continuous measures, while the Wilcoxon rank sum or Kruskal-Wallis tests were used for nonparametric variables. We used $\chi^{2}$ or Fisher exact tests for categorical variables.

We used an additive genetic model (i.e. copies of the minor allele or haplotype) for all logistic and linear regression analyses. All models were adjusted for age, sex, race, and treatment center. We also explored SNP-by-SNP interactions in all regression models.

Logistic regression analysis was used to examine the association of each PNMT gene polymorphism and haplotype, as well as urine catecholamine levels, with the outcomes of in-hospital death, dialysis requirement, and the presence of oliguria or hemodynamic shock. Urine output was log transformed due to a skewed distribution.

All statistical tests were performed with the use of SAS software, version 9.1 (SAS Institute, Cary, N.C., USA). A p $\leq 0.05$ was considered significant for all outcomes.

\section{Results}

Characteristics of the AKI (Case) and Control Cohort

A total of 961 Caucasian patients were included in the case-control study, of whom 194 were enrolled for a diagnosis of AKI. Of those with AKI, 82 (42\%) of patients required dialysis and 49 (25\%) died over a median followup of 16 days (interquartile range 9-26 days). The majority of subjects enrolled had severe AKI as defined by the
AKIN criteria. There were 64 (33\%) classified with stage 1, 32 (16\%) with stage 2, and 93 (48\%) with stage 3 AKI. Genotype analyses were performed on 194 subjects for the PNMT G-161A promoter SNP and 184 for the PNMT A $+1543 \mathrm{G}$ coding SNP. The minor allele frequency of the PNMT promoter -161 A allele was $54 \%$, but was only $3 \%$ for the coding $+1543 \mathrm{G}$ allele. Tests for Hardy-Weinberg equilibrium showed no deviation from expected frequencies (table 1).

Patients with AKI, on average, were 20 years older than the control subjects, and had a significantly higher prevalence of hypertension (78 vs. $19 \%$; $\mathrm{p}<0.001$ ). The genotype frequency of the PNMT G-161A SNP did not vary between patients with $A K I$ and control subjects. The $P N M T+1543 \mathrm{G}$ allele, however, was significantly associated with AKI, with an odds ratio (OR) of 2.19 [95\% confidence interval (CI) 1.04-4.60], when adjusted for differences in age, sex, and presence of hypertension (table 2).

The characteristics of the AKI cohort stratified by PNMT genotype are shown in table 3. Patient demographics and disease severity measures did not significantly differ across genotypes, except at position -161 , where the heterozygous (G/A) genotype was associated with a lower baseline serum creatinine compared to both homozygous genotypes $(\mathrm{p}=0.02)$. Urine adrenaline was significantly lower among patients with the PNMT $-161 \mathrm{~A} / \mathrm{A}$ genotype $(\mathrm{p}=0.04)$. The $P N M T+1543 \mathrm{~A} / \mathrm{A}$ genotype was only associated with a higher prevalence of hypertension ( 80 vs. $56 \%, \mathrm{p}=0.03$ ).

\section{PNMT Genotypes and AKI-Related Outcomes}

Each copy of the PNMT promoter -161 A allele was associated with $41 \%$ lower odds for in-hospital mortality, and this protective benefit persisted after multivariate adjustment (table 4). Similarly, there was a lower odds for hemodynamic shock in those carrying copies of the A allele (adjusted OR 0.63, 95\% CI: 0.40-1.00). Although the effect 
Table 2. Association of PNMT genotypes with the development of AKI

\begin{tabular}{lc}
\hline PNMT SNP & OR $(95 \% \mathrm{CI})$ \\
\hline $\begin{array}{l}\text {-161 A allele (vs. G/G genotype) }(\mathrm{n}=951) \\
\quad \text { Unadjusted }\end{array}$ & $0.81(0.65-1.03)$ \\
$\quad \begin{array}{l}\text { Adjusted for age, sex, and presence of } \\
\text { hypertension }\end{array}$ & $0.81(0.61-1.07)$ \\
$+\begin{array}{l}\text { 1543 G allele (vs. A/A genotype) }(\mathrm{n}=961) \\
\quad \text { Unadjusted } \\
\text { Adjusted for age, sex, and presence of } \\
\quad \text { hypertension }\end{array}$ & $1.38(0.76-2.49)$ \\
\hline${ }^{*} \mathrm{p} \leq 0.05$. & $2.19(1.04-4.60)^{*}$ \\
\hline
\end{tabular}

estimate was in the same direction towards a protective effect, there was no significant association with dialysis requirement or the development of oliguria (table 4).

The PNMT $+1543 \mathrm{G} / \mathrm{A}$ genotype was associated with a 3.3-fold increased odds for oliguria, before and after adjusting for age, sex and treatment center. This SNP, however, was not associated with in-hospital death, dialysis requirement, or presence of hemodynamic shock.

\section{PNMT Haplotypes and AKI-Related Outcomes}

The most common haplotype was $-161 \mathrm{G} /+1543 \mathrm{~A}$ (haplotype 1) estimated on 231 chromosomes, while $-161 \mathrm{~A} /+1543 \mathrm{~A}$ (haplotype 2) was detected on 199 chromosomes. The haplotype analyses revealed that haplotype 1 (increasing copies of $-161 \mathrm{G} /+1543 \mathrm{~A}$ ) was associated with a higher prevalence of hemodynamic shock (adjusted OR 1.56, 95\% CI: 1.00-2.44). Table 4 also shows that increasing copies of $-161 \mathrm{~A} /+1543 \mathrm{~A}$ (haplotype 2 ) was associated with decreased in-hospital mortality (adjusted OR 0.60, 95\% CI: 0.37-0.99) and less hemodynamic shock (adjusted OR 0.65, 95\% CI: 0.42-1.00). Haplotype 2 also trended towards an association with less oliguria (adjusted OR 0.63, 95\% CI: 0.37-1.08, p = 0.09).

We tested for SNP-by-SNP interactions, but found no significant associations with any of our outcomes (data not shown).

\section{Urine Catecholamine Levels and AKI-Related}

Outcomes

We examined the association of urine adrenaline, noradrenaline, and their ratio with adverse outcomes (table 5). On multivariate analysis, each log increase in urine adrenaline was significantly associated with an adjusted 1.5-fold increased odds for in-hospital mortality, and a 1.36-fold increased odds for the presence of hemodynamic shock. Similarly, on multivariate analysis, each log increase in urine noradrenaline was associated with 1.23fold increased odds for in-hospital mortality, and 1.32fold increased odds for the presence of hemodynamic shock. There was no association between urine catecholamine levels and dialysis requirement or the presence of oliguria. We also found no interactions between vasopressor use and urine catecholamine excretion with any of the outcomes (data not shown).

\section{Discussion}

In the present study, we examined the association of a promoter and synonymous coding SNP of the PNMT gene with the development of AKI in a Caucasian population. The PNMT coding $+1543 \mathrm{G}$ allele was associated with more than a 2-fold increase in the development of AKI. In those with AKI, we studied the association of PNMT SNPs with disease severity and in-hospital mortality. The PNMT promoter -161 A allele was strongly associated with lower odds for in-hospital mortality and hemodynamic shock, while the PNMT +1543 G/A genotype was associated with oliguria.

The PNMT polymorphisms that we examined included a promoter and a coding (albeit synonymous, Lys152Lys) SNP, both of which might influence gene expression. The PNMT promoter $-161 \mathrm{~A} / \mathrm{A}$ genotype was also associated with lower urine adrenaline levels. We also noted that higher urine adrenaline levels were associated with higher odds for in-hospital mortality. This relationship between genotype and intermediate phenotype supports the possibility of a functional role of the PNMT -161 promoter SNP.

We also conducted haplotype analyses to further refine our findings. Due to the low minor allele frequency of the PNMT +1543 SNP, the common haplotypes generated did not provide any additional information compared to the individual SNPs alone. This could also suggest that our candidate SNPs represent the functional polymorphism(s), and are not simply in the same haplotype block, i.e. close linkage disequilibrium, to another 'culprit' SNP. However, this clinical study does not establish a precise mechanistic link between PNMT gene expression and outcomes.

The adrenergic pathway has been studied in kidney disease, but there are limited genetic studies of these pathways in AKI. The catechol-O-methyltransferase (COMT) gene polymorphism (nonsynonymous G-to-A 
Table 3. Characteristics of patients with AKI stratified by PNMT genotypes

\begin{tabular}{|c|c|c|c|c|c|c|c|}
\hline \multirow[t]{2}{*}{ Characteristic } & \multicolumn{4}{|c|}{ PNMT promoter -161 G/A genotypes $(\mathrm{n}=184)$} & \multicolumn{3}{|c|}{$P N M T$ coding +1543 A/G genotypes $(\mathrm{n}=194)$} \\
\hline & $\mathrm{GG}(\mathrm{n}=41)$ & $\mathrm{GA}(\mathrm{n}=102)$ & $\mathrm{AA}(\mathrm{n}=41)$ & $\mathrm{p}$ value & $\mathrm{AA}(\mathrm{n}=178)$ & $\mathrm{AG}(\mathrm{n}=16)$ & $\mathrm{p}$ value \\
\hline Age, years & $67 \pm 15$ & $66 \pm 15$ & $62 \pm 16$ & 0.28 & $65 \pm 15$ & $68 \pm 13$ & 0.40 \\
\hline Male sex, n & $22(54)$ & $49(48)$ & $29(71)$ & 0.05 & $97(54)$ & $8(50)$ & 0.73 \\
\hline BMI & $33.4 \pm 17.5$ & $31.1 \pm 8.6$ & $30.9 \pm 8.3$ & 0.51 & $31.7 \pm 11.3$ & $32.5 \pm 9.4$ & 0.81 \\
\hline Contributing causes to AKI, $\mathrm{n}$ & & & & 0.27 & & & 0.22 \\
\hline Ischemic & $11(27)$ & $36(35)$ & $7(17)$ & & $50(28)$ & $6(38)$ & \\
\hline Nephrotoxic & $8(20)$ & $15(15)$ & $6(15)$ & & $30(17)$ & $0(0)$ & \\
\hline Septic & $6(15)$ & $7(7)$ & $5(12)$ & & $17(10)$ & $3(19)$ & \\
\hline Multifactorial/other & $16(39)$ & $44(43)$ & $23(56)$ & & $81(46)$ & $7(44)$ & \\
\hline \multicolumn{8}{|l|}{ Comorbid conditions, $\mathrm{n}$} \\
\hline Diabetes mellitus & $17(41)$ & $42(41)$ & $21(51)$ & 0.53 & $80(45)$ & $6(38)$ & 0.57 \\
\hline Cardiovascular disease & $33(80)$ & $67(66)$ & $28(68)$ & 0.22 & $124(70)$ & $11(69)$ & 0.94 \\
\hline Hypertension & $32(78)$ & $78(76)$ & $34(83)$ & 0.70 & $142(80)$ & $9(56)$ & 0.03 \\
\hline Liver cirrhosis & $3(7)$ & $12(12)$ & $2(5)$ & 0.39 & $14(8)$ & $3(19)$ & 0.14 \\
\hline Chronic lung disease & $9(22)$ & $17(17)$ & $5(12)$ & 0.50 & $29(16)$ & $4(25)$ & 0.37 \\
\hline Chronic kidney disease & $26(63)$ & $65(64)$ & $27(66)$ & 0.97 & $114(64)$ & $10(63)$ & 0.90 \\
\hline Medical (vs. surgical) admission, $\mathrm{n}$ & $27(66)$ & $70(69)$ & $28(68)$ & 0.95 & $121(68)$ & $11(69)$ & 0.95 \\
\hline ICU admission, $\mathrm{n}$ & $34(83)$ & $81(79)$ & $28(68)$ & 0.23 & $137(77)$ & $11(69)$ & 0.46 \\
\hline Presence of SIRS, $\mathrm{n}$ & $24(59)$ & $43(42)$ & $19(46)$ & 0.21 & $84(47)$ & $5(31)$ & 0.22 \\
\hline Enrollment MAP, mm Hg & $74.6 \pm 16.6$ & $74.2 \pm 19.0$ & $79.4 \pm 14.5$ & 0.26 & $75.9 \pm 17.5$ & $71.9 \pm 17.8$ & 0.39 \\
\hline \multicolumn{8}{|l|}{ Median serum creatinine, $\mu \mathrm{mol} / \mathrm{l}$} \\
\hline Baseline & $133(98-168)$ & $115(89-168)$ & $142(115-213)$ & 0.02 & $124(98-168)$ & $106(89-151)$ & 0.29 \\
\hline Enrollment & $257(204-346)$ & $284(195-390)$ & $284(195-470)$ & 0.46 & 275 (204-399) & $337(222-426)$ & 0.36 \\
\hline Peak & $337(248-434)$ & $337(239-443)$ & $364(231-550)$ & 0.78 & $346(231-479)$ & $319(248-443)$ & 0.96 \\
\hline Discharge & $177(115-222)$ & $168(115-266)$ & $204(133-372)$ & 0.23 & $177(124-275)$ & $177(106-257)$ & 0.79 \\
\hline AKI stage at enrollment, $\mathrm{n}$ & & & & 0.66 & & & 0.28 \\
\hline Stage 1 & $13(32)$ & $33(32)$ & $18(44)$ & & $61(34)$ & $3(19)$ & \\
\hline Stage 2 & $6(15)$ & $20(20)$ & $4(10)$ & & $27(15)$ & $5(31)$ & \\
\hline Stage 3 & $20(49)$ & $47(46)$ & $18(44)$ & & $85(48)$ & $8(50)$ & \\
\hline Hospital length of stay, days & $20(11-32)$ & $17(9-25)$ & $12(10-21)$ & 0.13 & $16(9-26)$ & $19(14-27)$ & 0.40 \\
\hline \multicolumn{8}{|l|}{ Median urine catecholamine level } \\
\hline Adrenaline, ng/mg creatinine & $6.8(3.4-10.7)$ & $6.7(3.1-13.4)$ & $3.5(1.7-7.7)$ & 0.04 & $5.9(2.7-11.7)$ & $7.1(3.9-10.7)$ & 0.59 \\
\hline Noradrenaline, ng/mg creatinine & $11.8(6.2-61.6)$ & $16.7(5.5-71.9)$ & $29.1(6.3-71.5)$ & 0.94 & $17.4(5.5-70.1)$ & $31.0(9.6-92.1)$ & 0.40 \\
\hline Adrenaline-noradrenaline ratio & $3.6(0.8-10.8)$ & $2.5(0.9-11.0)$ & $5.9(1.4-23.5)$ & 0.22 & $3.6(0.9-12.4)$ & $4.0(1.8-26.7)$ & 0.47 \\
\hline
\end{tabular}

Figures in parentheses represent percentage. BMI = Body mass index; ICU = intensive care unit; SIRS = systemic inflammatory response syndrome; $\mathrm{MAP}=$ mean arterial pressure.

Table 4. Association of PNMT genotypes and haplotype with adverse clinical outcomes in patients with AKI

\begin{tabular}{|c|c|c|c|c|}
\hline \multirow[t]{2}{*}{ PNMT SNP } & \multicolumn{4}{|l|}{ OR $(95 \% \mathrm{CI})$} \\
\hline & in-hospital death & dialysis requirement & oliguria & hemodynamic shock \\
\hline \multicolumn{5}{|l|}{-161 A allele (vs. G/G genotype) } \\
\hline Unadjusted & $0.59(0.35-0.99)^{*}$ & $0.82(0.53-1.27)$ & $0.68(0.39-1.18)$ & $0.65(0.41-1.03)$ \\
\hline Adjusted for age, sex and treatment center & $0.58(0.35-0.99)^{*}$ & $0.80(0.51-1.25)$ & $0.67(0.38-1.20)$ & $0.63(0.40-1.00)^{*}$ \\
\hline \multicolumn{5}{|l|}{ +1543 G/A genotype (vs. A/A genotype) } \\
\hline Unadjusted & $1.88(0.65-5.49)$ & $1.41(0.51-3.91)$ & $3.23(1.15-9.57)^{*}$ & $1.02(0.35-2.93)$ \\
\hline Adjusted for age, sex, and treatment center & $1.85(0.63-5.43)$ & $1.48(0.53-4.16)$ & $3.35(1.13-9.95)^{*}$ & $1.04(0.36-3.04)$ \\
\hline \multicolumn{5}{|l|}{$-161 \mathrm{~A} /+1543$ A haplotype } \\
\hline Unadjusted & $0.61(0.37-0.99)^{*}$ & $0.81(0.53-1.23)$ & $0.63(0.37-1.06)$ & $0.67(0.44-1.04)$ \\
\hline Adjusted for age, sex, and treatment center & $0.60(0.37-0.99)^{*}$ & $0.78(0.51-1.19)$ & $0.63(0.37-1.08)$ & $0.65(0.42-1.00)^{*}$ \\
\hline
\end{tabular}


Table 5. Association of urine catecholamine levels with adverse clinical outcomes in patients with AKI

\begin{tabular}{|c|c|c|c|c|}
\hline \multirow[t]{2}{*}{ Urine catecholamine } & \multicolumn{4}{|l|}{ OR (95\% CI) } \\
\hline & in-hospital death & dialysis requirement & oliguria & hemodynamic shock $^{1}$ \\
\hline \multicolumn{5}{|l|}{ Adrenaline (per ng/mg log increase) } \\
\hline Unadjusted & $1.48(1.07-2.04)^{*}$ & $1.17(0.89-1.54)$ & $1.00(0.70-1.42)$ & $1.33(1.00-1.77)^{*}$ \\
\hline $\begin{array}{l}\text { Adjusted for age, sex, treatment center, } \\
\text { and vasopressor use }\end{array}$ & $1.50(1.06-2.13)^{*}$ & $1.14(0.85-1.52)$ & $0.89(0.60-1.31)$ & $1.36(1.01-1.83)^{*}$ \\
\hline \multicolumn{5}{|l|}{ Noradrenaline (per ng/mg log increase) } \\
\hline Unadjusted & $1.27(1.04-1.55)^{*}$ & $1.11(0.93-1.32)$ & $1.14(0.92-1.42)$ & $1.32(1.09-1.58)^{*}$ \\
\hline $\begin{array}{l}\text { Adjusted for age, sex, treatment center, } \\
\text { and vasopressor use }\end{array}$ & $1.23(1.00-1.53)^{*}$ & $1.10(0.92-1.32)$ & $1.03(0.81-1.31)$ & $1.32(1.10-1.59)^{*}$ \\
\hline
\end{tabular}

${ }^{*} \mathrm{p} \leq 0.05 .{ }^{1}$ Model not adjusted for vasopressor use, as it is included in the definition of hemodynamic shock.

polymorphism in exon 4) was studied in 260 patients undergoing cardiac surgery. The COMT enzyme is essential for catecholamine degradation. Homozygosity for the minor allele, reflecting low COMT enzymatic activity, was found to be associated with a higher prevalence and longer duration of hemodynamic shock, incidence of AKI, and prolonged hospital stay [13]. A previous study by one of the coauthors examined adrenergic gene polymorphisms in patients with chronic kidney disease. These authors explored the gene variants in chromogranin A, an enzyme that regulates catecholamine storage and release and plays a role in influencing sympathetic tone, and showed an association with the risk of hypertensive kidney failure in black patients [14], while variants at several sites in the adrenergic pathway influenced urine albumin excretion [15]. We have also previously explored oxidative stress-related genes in AKI, and found that polymorphism in the nicotinamide adenine dinucleotide phosphate (NADPH) oxidase p22phox was associated with dialysis requirement or in-hospital death [16]. The adrenergic pathway has been proposed to be important in critical illness, but this had not previously been extended to AKI. Exploration of additional candidate genes associated with catecholamine biosynthesis, catabolism, exocytosis, catecholamine receptors, and postreceptor signal transduction would be appropriate.

Limitations of our study include the sample size, which, even with 194 subjects with AKI, may be less than optimal for a gene association study and which is a vexing issue in genetic analyses of complex diseases. Also, the PNMT A $+1543 \mathrm{G}$ polymorphism had a low minor allele frequency $(\sim 3 \%)$, enhancing the likelihood of a type II error for some of our associations. We included a spectrum of phenotypes likely to be influenced by adrenergic homeostasis, namely dialysis requirement, oliguria and hemodynamic shock, and considered these to be intermediate markers of mortality. We acknowledge that dialysis initiation and management of oliguria is subjective, and is influenced by patient as well as physician factors. Furthermore, we enrolled subjects with AKI at the time of nephrology consultation rather than at its onset. Finally, our findings are restricted to Caucasian subjects and cannot be generalized to other ethnic groups.

The strengths of our study include a substantial number of control subjects without AKI, which allowed us to examine whether the PNMT SNPs are associated with the development of AKI. For patients with AKI, we were able to prospectively and comprehensively capture in-hospital outcomes, without attrition or loss to follow-up. Our AKI population was seen and managed in 2 academic medical centers and we believe it is representative and generalizable to most tertiary care settings.

In conclusion, in the present study, we provide support for the hypothesis that PNMT gene polymorphisms play a role in characterizing the development of AKI as well as predicting clinical outcomes in patients with AKI. Specifically, PNMT SNPs are associated with in-hospital mortality, hemodynamic compromise, and adverse renal outcomes such as oliguria. We also found some correlation between the PNMT promoter G-161A SNP and urine adrenaline levels, suggesting a possible functional role for this polymorphism. This relationship should be further explored, and externally validated in other more diverse populations. Adrenergic gene pathways may provide valuable genomic biomarkers for the development of AKI, risk stratification, prognosis, and possibly response to therapeutic interventions. 


\section{Acknowledgments}

The authors would like to acknowledge the assistance of the UAB-UCSD O'Brien Core Center for Acute Kidney Injury Research (NIH P30 DK079337) for the genomic analyses, Dr. Anupam Agarwal, Dr. Ravindra L. Mehta, and Dr. Inga Peter for their critical reviews in the preparation of this manuscript, and Robert W. MacKinnon, RN, for assistance with enrollment of study participants.
This study was supported in part by grants from the National Institutes of Health (DK065102 and DK077751 to B.L.J.; HL58120 and MD000220 to D.T.O.). A.A. was supported by a Biomedical Fellowship Award from the Kidney Foundation of Canada, and O.L. by a grant from the American Heart Association.

\section{References}

1 Nash K, Hafeez A, Hou S: Hospital-acquired renal insufficiency. Am J Kidney Dis 2002; 39:930-936.

-2 Mehta RL, Pascual MT, Soroko S, Savage BR, Himmelfarb J, Ikizler TA, Paganini EP, Chertow GM: Spectrum of acute renal failure in the intensive care unit: the Picard experience. Kidney Int 2004;66:1613-1621.

-3 Silvester W, Bellomo R, Cole L: Epidemiology, management, and outcome of severe acute renal failure of critical illness in Australia. Crit Care Med 2001;29:1910-1915.

4 Wong DL: Epinephrine biosynthesis: hormonal and neural control during stress. Cell Mol Neurobiol 2006;26:891-900.

5 Axelrod J, Reisine TD: Stress hormones: their interaction and regulation. Science 1984;224:452-459.

-6 Goldstein DS, Eisenhofer G, Kopin IJ: Clinical catecholamine neurochemistry: a legacy of Julius Axelrod. Cell Mol Neurobiol 2006; 26:695-702.

-7 Hou SH, Bushinsky DA, Wish JB, Cohen JJ, Harrington JT: Hospital-acquired renal insufficiency: a prospective study. Am J Med 1983;74:243-248.
8 Mehta RL, Kellum JA, Shah SV, Molitoris BA, Ronco C, Warnock DG, Levin A: Acute Kidney Injury Network: report of an initiative to improve outcomes in acute kidney injury. Crit Care 2007;11:R31.

-9 Levy MM, Fink MP, Marshall JC, Abraham E, Angus D, Cook D, Cohen J, Opal SM, Vincent JL, Ramsay G: 2001 SCCM/ESICM/ ACCP/ATS/SIS International Sepsis Definitions Conference. Crit Care Med 2003;31 1250-1256.

10 Ronaghi M: Pyrosequencing sheds light on DNA sequencing. Genome Res 2001;11:311.

11 Sasaoka T, Kaneda N, Kurosawa Y, Fujuta K, Nagatsu T: Structure of human phenylethanolamine N-methytransferase gene: existence of two types of mRNA with different transcription initiation sites. Neurochem Int 1989;15:555-565.

12 Halperin E, Eskin E: Haplotype reconstruction from genotype data using imperfect phylogeny. Bioinformatics 2004;20:18421849.

13 Haase-Fielitz A, Haase M, Bellomo R, Lambert G, Matalanis G, Story D, Doolan L, Buxton B, Gutteridge G, Luft FC, Schunck WH, Dragun D: Decreased catecholamine degradation associates with shock and kidney injury after cardiac surgery. J Am Soc Nephrol 2009;20:1393-1403
14 Salem RM, Cadman PE, Chen Y, Rao F, Wen G, Hamilton BA, Rana BK, Smith DW, Stridsberg M, Ward HJ, Mahata M, Mahata SK, Bowden DW, Hicks PJ, Freedman BI, Schork NJ, O'Connor DT: Chromogranin a polymorphisms are associated with hypertensive renal disease. J Am Soc Nephrol 2008; 19:600-614.

15 Rao F, Wessel J, Wen G, Zhang L, Rana BK, Kennedy BP, Greenwood TA, Salem RM, Chen Y, Khandrika S, Hamilton BA, Smith DW, Holstein-Rathlou NH, Ziegler MG, Schork NJ, O'Connor DT: Renal albumin excretion: twin studies identify influences of heredity, environment, and adrenergic pathway polymorphism. Hypertension 2007;49: 1015-1031.

16 Perianayagam MC, Liangos O, Kolyada AY, Wald R, MacKinnon RW, Li L, Rao M, Balakrishnan VS, Bonventre JV, Pereira BJ, Jaber BL: NADPH oxidase p22phox and catalase gene variants are associated with biomarkers of oxidative stress and adverse outcomes in acute renal failure. J Am Soc Nephrol 2007;18:255-263. 